\title{
DNA-Gold Nanozyme-Modified Paper Device for Enhanced Colorimetric Detection of Mercury Ions
}

\author{
Min-Xin Mao ${ }^{1,2,+}$, Rong Zheng ${ }^{2,+}$, Chi-Fang Peng ${ }^{1,2, * \mathbb{C}}$ and Xin-Lin Wei ${ }^{3}$ \\ 1 State Key Laboratory of Dairy Biotechnology, Shanghai Engineering Research Center of Dairy Biotechnology, \\ Dairy Research Institute, Bright Dairy \& Food Co., Ltd., Shanghai 200436, China; \\ 6190112080@jiangnan.edu.cn \\ 2 School of Food Science and Technology, Jiangnan University, Wuxi 214122, China; \\ 6170112120@stu.jiangnan.edu.cn \\ 3 School of Agriculture and Biology, Shanghai Jiaotong University, Shanghai 200240, China; \\ weixinlin@sjtu.edu.cn \\ * Correspondence: pcf@jiangnan.edu.cn \\ $\dagger$ These authors contributed equally to this work.
}

Received: 9 December 2020; Accepted: 16 December 2020; Published: 18 December 2020

check for updates

\begin{abstract}
In this work, a paper device consisted of a patterned paper chip, wicking pads, and a base was fabricated. On the paper chip, DNA-gold nanoparticles (DNA-AuNPs) were deposited and $\mathrm{Hg}^{2+}$ ions could be adsorbed by the DNA-AuNPs. The formed DNA-AuNP/ $\mathrm{Hg}^{2+}$ nanozyme could catalyze the tetramethylbenzidine (TMB) $-\mathrm{H}_{2} \mathrm{O}_{2}$ chromogenic reaction. Due to the wicking pads, a larger volume of $\mathrm{Hg}^{2+}$ sample could be applied to the paper device for $\mathrm{Hg}^{2+}$ detection and therefore the color response could be enhanced. The paper device achieved a cut-off value of $50 \mathrm{nM}$ by the naked eye for $\mathrm{Hg}^{2+}$ under optimized conditions. Moreover, quantitative measurements could be implemented by using a desktop scanner and extracting grayscale values. A linear range of $50-2000 \mathrm{nM} \mathrm{Hg}^{2+}$ was obtained with a detection limit of $10 \mathrm{nM}$. In addition, the paper device could be applied in the detection of environmental water samples with high recoveries ranging from $85.7 \%$ to $105.6 \%$. The paper-device-based colorimetric detection was low-cost, simple, and demonstrated high potential in real-sample applications.
\end{abstract}

Keywords: paper device; signal enhancement; mercury ion; colorimetric detection

\section{Introduction}

Mercury ions $\left(\mathrm{Hg}^{2+}\right)$ are one of toxic heavy metals. They are widely found in the environment [1], are a serious threat to human health [2]. In order to control the risk of $\mathrm{Hg}^{2+}$, the US Environmental Protection Agency (EPA) and the World Health Organization (WHO) set a maximum contents of $\mathrm{Hg}^{2+}$ in drinking water which are $2.0 \mu \mathrm{g} / \mathrm{L}(10 \mathrm{nM})$ and $6.0 \mu \mathrm{g} / \mathrm{L}(30 \mathrm{nM})$, respectively [3]. In practice, numerous conventional lab-dependent techniques such as inductively-coupled plasma mass spectrometry (ICP-MS) [4], atomic fluorescence spectrometry (AFS) [5], and high performance liquid chromatography (HPLC) [6,7] have been well-established for detection of $\mathrm{Hg}^{2+}$. However, their operations are highly dependent on time-consuming sample pretreatments, expensive instrumentation, and skilled technicians, making them unsuitable for rapid and on-site detection of target $\mathrm{Hg}^{2+}$ ions $[8,9]$.

In recent years, many researchers have established a large number of methods for the detection of $\mathrm{Hg}^{2+}$ based on nanomaterials, such as fluorescent [10], colorimetric [11], chemiluminescent [12], surface-enhanced raman spectroscopy (SERS), and electrochemical methods [13,14]. The above methods have demonstrated many advantages, such as rapidness and high sensitivity. $\mathrm{Hg}^{2+}$ can strongly interact with many nanomaterials such as gold nanoparticles (AuNPs), gold nanorods, 
and silver nanoparticles. When these nanoparticles adsorb $\mathrm{Hg}^{2+}, \mathrm{Au}-\mathrm{Hg}$ nano-alloys or $\mathrm{Ag}-\mathrm{Hg}$ nano-alloys could be formed [15]. Some researchers have reported that $\mathrm{Au}-\mathrm{Hg}$ nano-alloys possess peroxidase-like property and could catalyze $\mathrm{H}_{2} \mathrm{O}_{2}$-mediated oxidation of tetramethylbenzidine (TMB) [16]. We reported that the DNA-AuNP complex could capture $\mathrm{Hg}^{2+}$ and form DNA-Au-Hg nano-alloys [17]. These DNA-Au-Hg nano-alloys demonstrated much stable peroxidase-like activity and could achieve highly-sensitive colorimetric detection of $\mathrm{Hg}^{2+}$.

Since Whiteside's group first proposed a paper-based device for the detection of biochemicals in blood, this device has received considerable attention by many researchers $[18,19]$. Paper is an excellent substrate material for sample filtration and preconcentration [20,21] due to its high surface-to-volume ratio, low-cost, and portability [22,23]. It has also been widely used in medical diagnosis [24,25], environmental monitoring [26], and food quality analysis [27], etc. Paper can be modified by various nanomaterials, such as ceria nanoparticles [28], AuNPs [29], silver nanoparticles [30,31], and carbon nanotubes [32-34] in order to develop assays for various targets or improve colorimetric homogeneity and intensity. For example, He et al. successfully developed an ultrasensitive nucleic acid biosensor based on HRP-AuNP dual labels and a lateral flow strip biosensor [35]. Qiao et al. developed a fluorometric $\mathrm{Hg}^{2+}$ test strip using $\mathrm{Au}-\mathrm{Ag}$ nanoclusters as fluorescent probes combined with suppressing "coffee stains" by a bio-inspired fabrication strategy [36]. Zhang et al. used Cy5-labeled functional ssDNA toward multiple analytes, graphene oxide, and paper substrate to fabricate a paper device to report the presence of the $\mathrm{Hg}^{2+}$ and $\mathrm{Ag}^{+}$ions and aminoglycoside antibiotics in food [37]. Li et al. prepared three kinds of doped carbon quantum dots and fabricated a smartphone-based three-channel ratio fluorescence device for simultaneous determination of $\mathrm{Hg}^{2+}, \mathrm{Fe}^{3+}$, and $\mathrm{Cu}^{2+}$ ions in environmental samples [38]. Zhou et al. developed a rapid and sensitive paper-based analytical device (PAD) to detect the total tetracyclines in environmental water based on a paper channel by field amplification stacking and fluorescent imaging [39].

In this paper, DNA-AuNPs were deposited onto filter paper and a nanozyme-based colorimetric detection of $\mathrm{Hg}^{2+}$ was carefully optimized on the filter paper. The detection was eventually carried out on a paper chip, which had detection zones modified with DNA-AuNPs and connected to a substrate reservoir by multiple channels [40]. Layers of filter paper as a wicking pad were placed under the detection zones to facilitate $\mathrm{Hg}^{2+}$ enrichment. This paper device demonstrated advantages including being simple, low-cost, and sensitive.

\section{Experimental}

\subsection{Reagents and Instruments}

Chloroauric acid $\left(\mathrm{HAuCl}_{4}\right)$ and sodium citrate were purchased from Sigma-Aldrich (Shanghai, China) and 3,3',5,5'-tetramethylbenzidine (TMB) and hydrogen peroxide were purchased from Aladdin Reagent Company (Shanghai, China). All metal ion standard solutions were purchased from the National Institute of Metrology P. R. China. All other reagents were of analytical grade. Whatman No. 1 filter paper was obtained from GE Healthcare (Shanghai, China). Ultra-pure water was prepared with a Milli-Q pure system for all the experiments.

UV-visible (UV-vis) absorption spectra were measured with an Agilent Cary $60 \mathrm{UV}$-vis spectrophotometer (Crawford Scientific, Strathaven, UK) at room temperature. Absorption values of reaction solutions were obtained with a microplate reader (Bio-Tek, Elx800, Winooski, VT, USA). Transmission electron microscopy (TEM) images were obtained on a JEOL JEM-2100 at an accelerating voltage of $200 \mathrm{kV}$.

\subsection{Fabrication of Gold Nanozyme Paper Device}

The pattern of paper chip was designed using CorelDRAW software. As shown in Figure S1a, the paper chip had a substrate reservoir, which connected with eight detection zones through eight 
channels. The pattern of the paper device base was similar to the pattern of the paper chip, except for having eight smaller holes located at the center of each detection zone (Figure 1).

The filter paper was cut into the paper chip according to the designed pattern by a $\mathrm{CO}_{2}$ laser engraving machine (Golden, CO 80403 USA). Then, the obtained paper cuttings were immersed into ultrapure water, rinsed for $30 \mathrm{~s}$, and then dried at $40{ }^{\circ} \mathrm{C}$ for later use.

A wood board was engraved by the $\mathrm{CO}_{2}$ laser to produce the pattern (Figure S1b). The depth of the groove for paper chip was set at $1.5 \mathrm{~mm}$ and other sizes are shown in the pattern. The engraved wood board was immersed in 1\% paraffin solution (dissolved in n-hexane) for $5 \mathrm{~min}$, and then baked at $80^{\circ} \mathrm{C}$ for $10 \mathrm{~min}$. The fabrication of the paper device is shown in Figure 1. Firstly, Scotch tape was attached to back side of the pretreated wood base. Layers of round filter paper as a wicking pad were filled into the holes of the wood board. Then the paper chip was fixed closely to the patterned wood board. The images of the paper device are shown in Figure S2.

\subsection{Colorimetric Detection of $\mathrm{Hg}^{2+}$ on Paper Device}

The DNA-AuNP complex were prepared according to our previous reports [17,41]. To each detection zone on paper chip, $2 \mu \mathrm{L}$ of the DNA-AuNPs $(0.6 \mathrm{nM})$ was added. After being dried at room temperature for $5 \mathrm{~min}, 20-100 \mu \mathrm{L}$ of standard $\mathrm{Hg}^{2+}$ solution or sample was added to each sample detection zone. After being incubated for $20 \mathrm{~min}, 300 \mu \mathrm{L}$ of substrate $\left(0.4 \mathrm{mM} \mathrm{TMB}\right.$ and $3.0 \% \mathrm{H}_{2} \mathrm{O}_{2}$ in $0.1 \mathrm{M}$ citric buffer) was added to the reagent reservoir. After the substrates were distributed to each detection zone, chromogenic reaction was initiated and continued for $20 \mathrm{~min}$. The color development was recorded by mobile phone and desktop scanning, and the colorimetric signal was analyzed using Image J software.

\subsection{Validation of the Colorimetric Detection}

Tap water and lake water samples from Li Lake (Wuxi, China) were spiked with different concentrations $(200,500$, and $1000 \mathrm{nM})$ of $\mathrm{Hg}^{2+}$, filtered twice through $0.22 \mu \mathrm{m}$ membrane, and then measured by the paper device. The lake water samples were filtered with filter paper modified with graphene oxide, and then filtered with a $0.22 \mu \mathrm{m}$ membrane to carry out the next detection.

\section{Results and Discussion}

\subsection{Fabrication of Paper Device}

Generally, the sensitivity of paper-based assays is negatively affected by small volumes of sample loaded onto a small-size detection zone [20,35,42]. The volume of loaded sample could be increased significantly through adopting water adsorbent, thereby improving the detection sensitivity [22]. In order to achieve enhanced sensitivity in our designed paper chip for $\mathrm{Hg}^{2+}$ colorimetric detection, we used a base to hold the paper chip and wicking pad. The patterns of paper chip and wood base were both easily produced. The cost of one paper chip is about 15 cents (CNY). The cost of the wood base is about twenty cents and it could be reusable. Thus, the device is low-cost. The wood base and paper chip were obtained through laser engraving as shown in Figure 1. The size of the paper chip and wood base could be easily controlled to match with each other. On the wood base, it was much simpler to cut a hole than engrave a well. In order to fix the wicking pad, scotch tape was used to seal the holes.

In order to prevent rapid sample leakage along the wood base surface, the inner surface of the base was hydrophobically modified by coating with paraffin. As shown in Figure S3, the contact angle to the waterdrop on the wood board surface was over 90 degree and the water drop on the base surface could be kept stable for over $60 \mathrm{~min}$. These results confirmed the good hydrophobicity of the paraffin-modified base, which facilitated stable sample flow vertical from paper chip to the wicking pad and $\mathrm{Hg}^{2+}$ absorption by the DNA-AuNPs on the detection zone (Figure 2). 


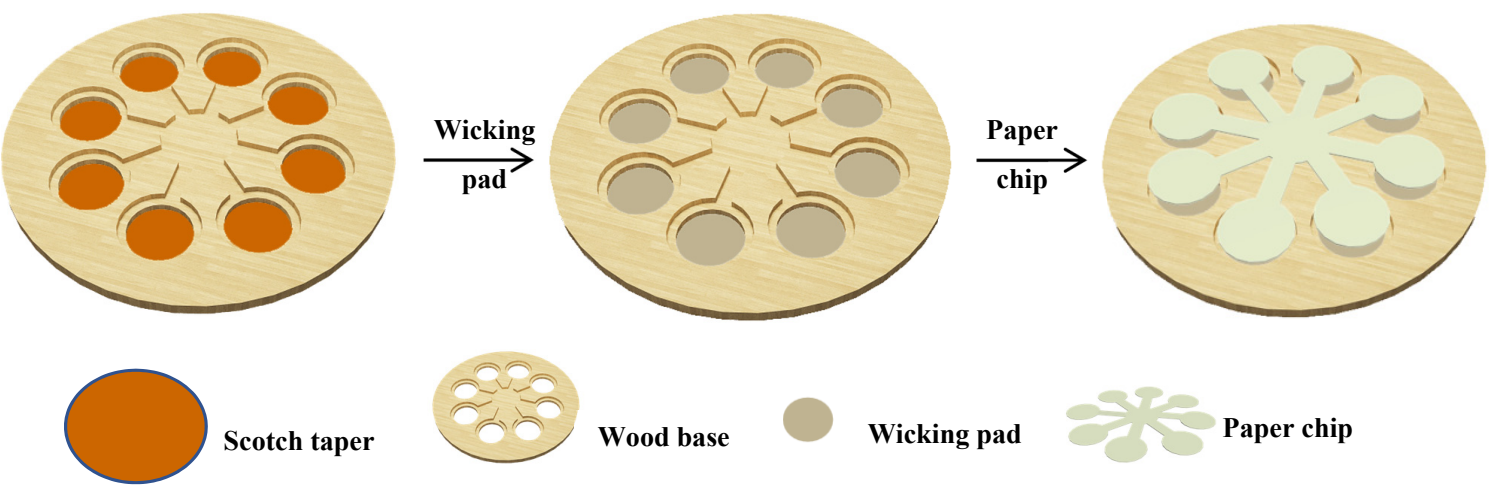

Figure 1. Schematic of paper device fabrication.

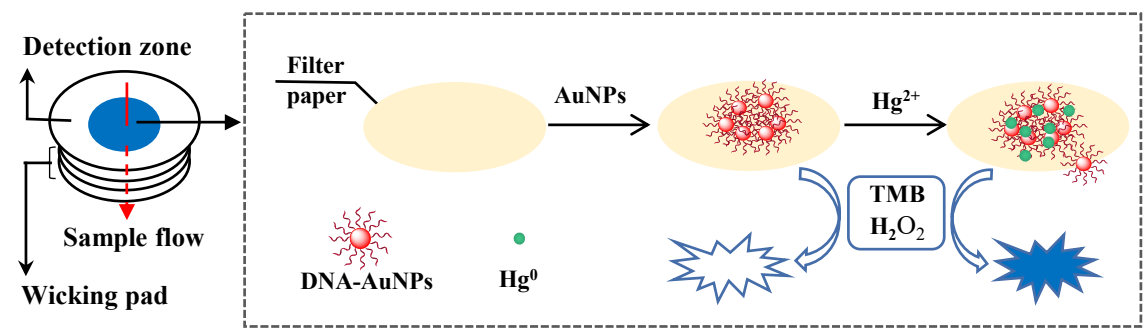

Figure 2. Schematic diagram of $\mathrm{Hg}^{2+}$ detection.

\subsection{Colorimetric Detection of $\mathrm{Hg}^{2+}$}

The DNA-AuNPs had peroxidase-like activity and could catalyze the chromogenic reaction of TMB- $\mathrm{H}_{2} \mathrm{O}_{2}$, but the catalytic activity was weak. The peroxidase activity of the DNA-AuNPs could be significantly enhanced after the DNA-AuNPs adsorbed $\mathrm{Hg}^{2+}$ [15] and produced a very strong peak of TMB- $\mathrm{H}_{2} \mathrm{O}_{2}$ at $650 \mathrm{~nm}$ (Figure S4).

In order to obtain sensitive detection on the paper chip, the effect of $\mathrm{H}_{2} \mathrm{O}_{2}$ concentration and DNA-AuNP concentration were investigated. The optimal conditions were evaluated by the colorimetric intensity difference, $\Delta \mathrm{I}=\mathrm{I}-\mathrm{I}_{0}$ ( $\mathrm{I}$ and $\mathrm{I}_{0}$ refer to the gray value obtained with and without $\mathrm{Hg}^{2+}$ ). As shown in Figure $3 a, b$, the highest color intensity could be obtained with $3 \% \mathrm{H}_{2} \mathrm{O}_{2}$ and $0.6 n M$ DNA-AuNPs, respectively.

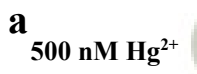

Blank
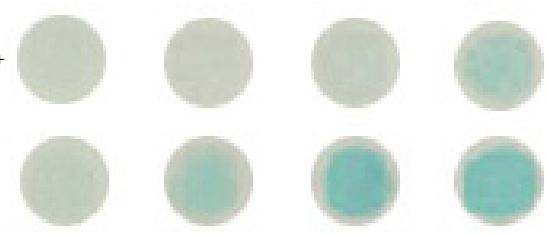

b
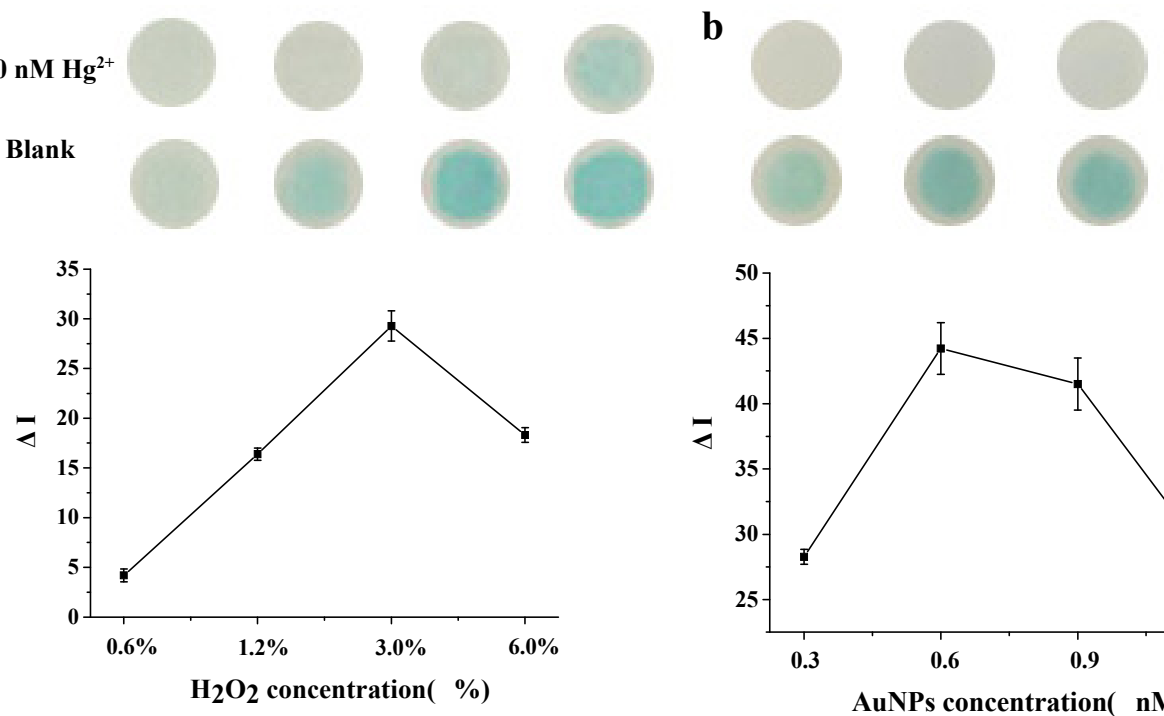
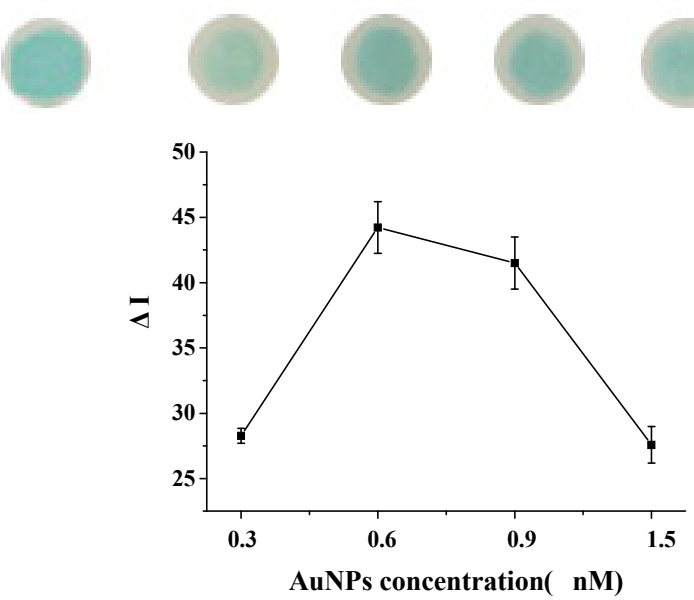

Figure 3. Optimization of $\mathrm{H}_{2} \mathrm{O}_{2}$ and DNA-AuNP concentration. (a) Effect of $\mathrm{H}_{2} \mathrm{O}_{2}$ concentration and (b) effect of DNA-AuNP concentration. 
The $\mathrm{Hg}^{2+}$ volume was also investigated. When more than $20 \mu \mathrm{L}$ was applied onto the detection zone, $\mathrm{Hg}^{2+}$ solution would overflow to the substrate reservoir, resulting in uncontrolled color development. With the superimposed wicking pad under paper chip, the volume of $\mathrm{Hg}^{2+}$ solution could be increased linearly with the increasing layers of wicking pad. In order to simplify the operation, five layers of wicking pad and $100 \mu \mathrm{L}$ of $\mathrm{Hg}^{2+}$ solution at most were investigated. As shown in Figure $4 \mathrm{a}$, it was found that darkest blue appeared when $60 \mu \mathrm{L}$ of $\mathrm{Hg}^{2+}$ solution was used. Compared with $20 \mu \mathrm{L}$ of $\mathrm{Hg}^{2+}$ solution, $60 \mu \mathrm{L}$ was suitable for paper chip alone, and the $\Delta \mathrm{I}$ increased four-fold. Unfortunately, no higher signal increasement was found when over $60 \mu \mathrm{L}$ of $\mathrm{Hg}^{2+}$ solution was dropped onto the paper chip. These results were probably due to the fact that part of the DNA-AuNPs could be washed away by excessive $\mathrm{Hg}^{2+}$ solution. After incubating with $\mathrm{Hg}^{2+}$ for $15-20 \mathrm{~min}$, the highest colorimetric intensity could be obtained when $60 \mu \mathrm{L}$ of $\mathrm{Hg}^{2+}$ solution was used (Figure $4 \mathrm{~b}$ ).
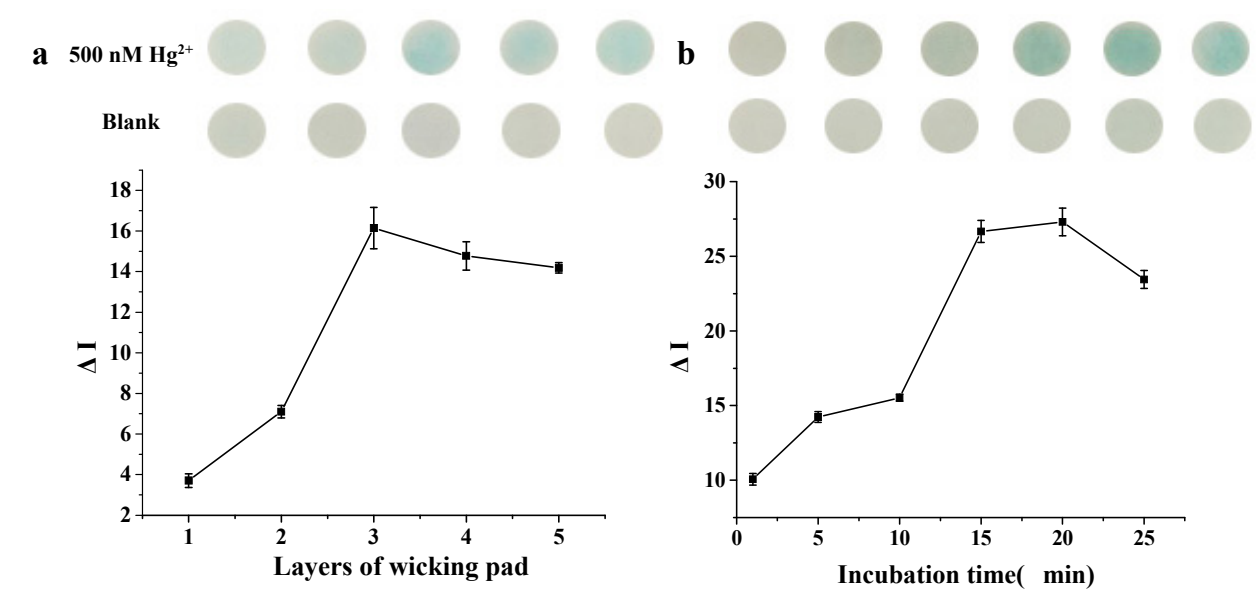

Figure 4. Optimization of the $\mathrm{Hg}^{2+}$ volume and adsorption time. (a) Effect of layers of wicking pad and (b) effect of incubation time.

As shown in Figure 5, the color intensity increased with the increased $\mathrm{Hg}^{2+}$ concentration on the paper device, and $50 \mathrm{nM} \mathrm{Hg}^{2+}$ could be distinguished by the naked eye. With the desktop scanning, quantitative determination could be implemented. A linear relationship between the gray intensity and logarithm of $\mathrm{Hg}^{2+}$ concentration could be obtained in the range of $0.05-2 \mu \mathrm{M}$. A detection limit of $10 \mathrm{nM}$ was achieved, based on a $3 \sigma /$ slope, where $\sigma$ was the standard deviation of blank samples. Compared with some typical nanomaterial-modified papers or test strips for $\mathrm{Hg}^{2+}$ colorimetric detection, the above paper-device-based detection demonstrated comparable sensitivity (Table S1).
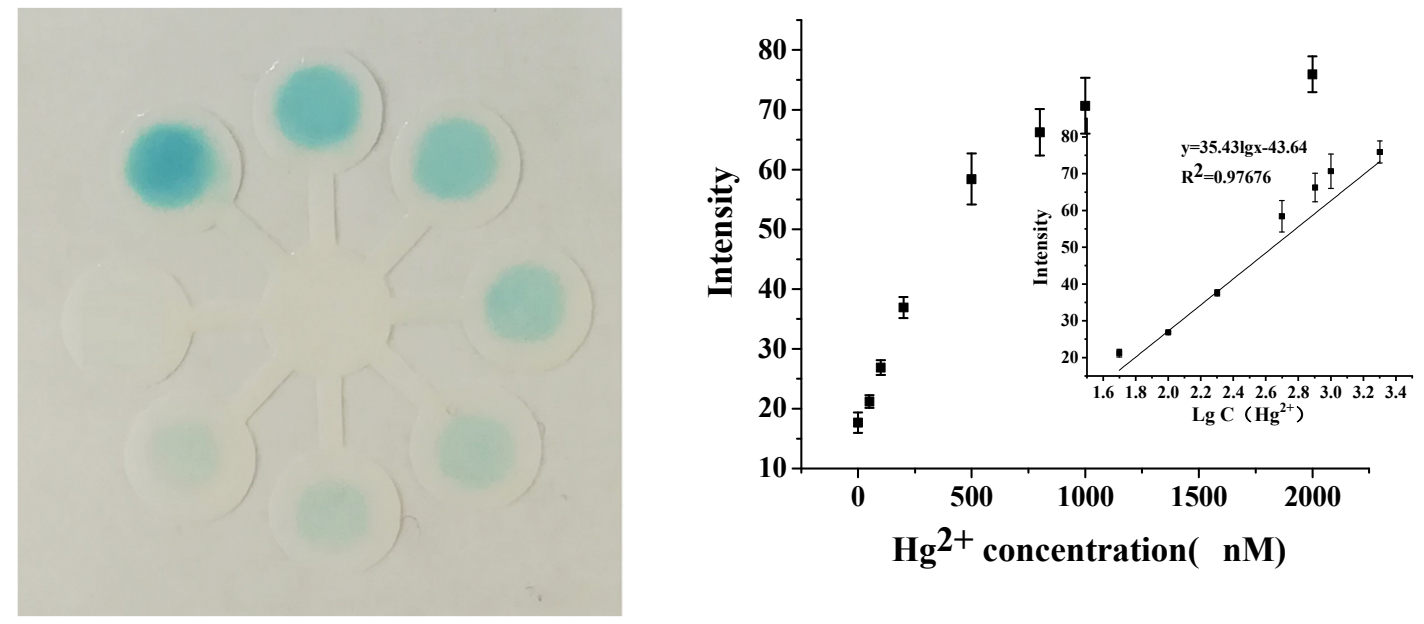

Figure 5. The image of detection of $\mathrm{Hg}^{2+}$ and calibration curve of colorimetric detection. 
To explore the selectivity of this colorimetric detection, various common metal ions including $\mathrm{MeHg}^{+}, \mathrm{Mn}^{2+}, \mathrm{Cu}^{2+}, \mathrm{Ni}^{2+}, \mathrm{Ba}^{2+}, \mathrm{Cd}^{2+}, \mathrm{Al}^{3+}, \mathrm{Zn}^{2+}, \mathrm{Fe}^{3+}, \mathrm{Cr}^{3+}, \mathrm{Co}^{2+}, \mathrm{Sr}^{2+}$, and $\mathrm{Bi}^{3+}$ were tested. As shown in Figure 6, $\mathrm{Hg}^{2+}$ ions $(1 \mu \mathrm{M})$ showed a deep blue color in the paper and negligible color responses were observed toward the other metal ions $(10 \mu \mathrm{M})$, indicating that the high selectivity of this method was toward $\mathrm{Hg}^{2+}$.

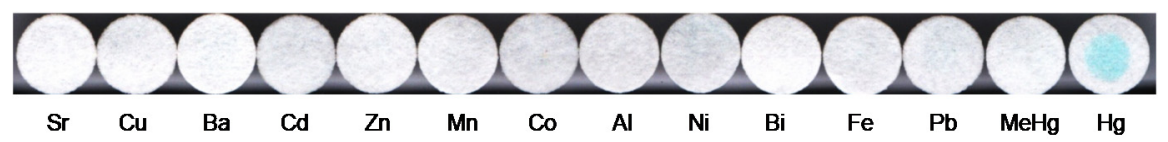

Figure 6. Selectivity of the method toward heavy metal ions.

\subsection{Application in Real Samples}

To verify the feasibility of this paper device in detecting real samples, tap water and lake water samples were spiked with $\mathrm{Hg}^{2+}$ and applied to the paper device. The results were obtained as shown in Table 1. The recoveries ranged from $85.7 \%$ to $105.6 \%$ when water samples spiked with 200, 500, and $1000 \mathrm{nM} \mathrm{Hg}^{2+}$ were measured. These results showed the great potential of this paper device for $\mathrm{Hg}^{2+}$ detection in practical applications.

Table 1. Determination of $\mathrm{Hg}^{2+}$ in tap water and lake water samples. $(\mathrm{n}=3)$.

\begin{tabular}{ccccc}
\hline Sample & Added (nM) & Detected (nM) & Recovery (\%) & RSD (\%) \\
\hline \multirow{3}{*}{ Tap water } & 200 & 189.8 & 94.9 & 3.6 \\
& 500 & 506.8 & 101.4 & 2.9 \\
& 1000 & 856.9 & 85.7 & 2.0 \\
Lake water & 200 & 197.6 & 98.8 & 4.7 \\
& 500 & 483.7 & 96.7 & 4.2 \\
& 1000 & 1056.0 & 105.60 & 2.7 \\
\hline
\end{tabular}

\section{Conclusions}

In conclusion, a paper device consisting of a patterned paper chip and a base were successfully fabricated. The designed paper chip and wicking pad on the paper device facilitated the operation of DNA-gold nanozyme-based colorimetric detection of $\mathrm{Hg}^{2+}$ and enhanced the sensitivity. The color development of $50 \mathrm{nM} \mathrm{Hg}^{2+}$ on the paper device could be distinguished by the naked eye. Moreover, quantitative analysis of the color could be implemented by desktop scanner and gray intensity extracting. The colorimetric detection of $\mathrm{Hg}^{2+}$ was a low-cost, simple operation that demonstrated great potential in real sample detection. In addition, the paper device could be extended to combine with other nanosensors for more applications.

Supplementary Materials: The following are available online at http://www.mdpi.com/2079-6374/10/12/211/s1, Figure S1: Designed pattern of (a) paper chip and (b) base of the paper device; Figure S2: Photographs of paper-based device. (a) paper chip; (b) wood base; (c) wood base filled with wicking pad and (d) paper device; Figure S3: Photograph of a water drop on wood board surface; Figure S4: UV- vis absorption spectra of $\mathrm{TMB}^{-} \mathrm{H}_{2} \mathrm{O}_{2}$ reaction. (a) DNA-AuNPs without $\mathrm{Hg}^{2+}$; (b) DNA-AuNPs with $\mathrm{Hg}^{2+}$. Table S1: Comparison of paper-based devices for the detection of $\mathrm{Hg}^{2+}$ reported in literatures.

Author Contributions: Investigation, manuscript writing (M.-X.M.); Investigation, data treatment (R.Z.); Experiment design, result discussion, manuscript revision (C.-F.P.); Result discussion, manuscript revision (X.-L.W.). All authors have read and agreed to the published version of the manuscript.

Funding: This research was funded by the MOST, PRC (National Key Research and Development Program 2018YFC1604400), the Open Project Program of State Key Laboratory of Dairy Biotechnology, Bright Dairy \& Food Co. Ltd. (SKLDB2017-00) and the National Natural Science Foundation of China (31871879).

Conflicts of Interest: The authors declare no conflict of interest. 


\section{References}

1. Li, X.; Zhang, Y.; Chang, Y.; Xue, B.; Kong, X.; Chen, W. Catalysis-reduction strategy for sensing inorganic and organic mercury based on gold nanoparticles. Biosens. Bioelectron. 2017, 92, 328-334. [CrossRef] [PubMed]

2. Deng, L.; Li, Y.; Yan, X.; Xiao, J.; Ma, C.; Zheng, J.; Liu, S.; Yang, R. Ultrasensitive and Highly Selective Detection of Bioaccumulation of Methyl-Mercury in Fish Samples via Ag-0/Hg-0 Amalgamation. Anal. Chem. 2015, 87, 2452-2458. [CrossRef] [PubMed]

3. Kan, C.; Shao, X.; Song, F.; Xu, J.; Zhu, J.; Du, L. Bioimaging of a fluorescence rhodamine-based probe for reversible detection of $\mathrm{Hg}$ (II) and its application in real water environment. Microchem. J. 2019, 150, 104142. [CrossRef]

4. Rofouei, M.K.; Rezaei, A.; Masteri-Farahani, M.; Khani, H. Selective extraction and preconcentration of ultra-trace level of mercury ions in water and fish samples using Fe3O4-magnetite-nanoparticles functionalized by triazene compound prior to its determination by inductively coupled plasma-optical emission spectrometry. Anal. Methods 2012, 4, 959-966.

5. Carneado, S.; Pero-Gascon, R.; Ibanez-Palomino, C.; Lopez-Sanchez, J.F.; Sahuquillo, A. Mercury(II) and methylmercury determination in water by liquid chromatography hyphenated to cold vapour atomic fluorescence spectrometry after online short-column preconcentration. Anal. Methods 2015, 7, 2699-2706. [CrossRef]

6. Zhou, Q.; Xing, A.; Zhao, K. Simultaneous determination of nickel, cobalt and mercury ions in water samples by solid phase extraction using multiwalled carbon nanotubes as adsorbent after chelating with sodium diethyldithiocarbamate prior to high performance liquid chromatography. J. Chromatogr. A 2014, 1360, 76-81. [CrossRef]

7. Wang, L.; Zhou, J.-B.; Wang, X.; Wang, Z.-H.; Zhao, R.-S. Simultaneous determination of copper, cobalt, and mercury ions in water samples by solid-phase extraction using carbon nanotube sponges as adsorbent after chelating with sodium diethyldithiocarbamate prior to high performance liquid chromatography. Anal. Bioanal. Chem. 2016, 408, 4445-4453. [CrossRef]

8. Anand, T.; Sankar, M. A dual colorimetric chemosensor for $\mathrm{Hg}(\mathrm{II})$ and cyanide ions in aqueous media based on a nitrobenzoxadiazole (NBD)-antipyrine conjugate with INHIBIT logic gate behaviour. Anal. Methods 2020, 12, 4526-4533. [CrossRef]

9. Feng, X.; Zhang, J.; Wang, J.; Han, A.; Fang, G.; Liu, J.; Wang, S. The stabilization of fluorescent copper nanoclusters by dialdehyde cellulose and their use in mercury ion sensing. Anal. Methods 2020, 12, 3130-3136. [CrossRef]

10. Chen, L.; Fu, X.; Lu, W.; Chen, L. Highly Sensitive and Selective Colorimetric Sensing of $\mathrm{Hg}^{2+}$ Based on the Morphology Transition of Silver Nanoprisms. ACS Appl. Mater. Interfaces 2013, 5, 284-290. [CrossRef]

11. Long, Y.J.; Li, Y.F.; Liu, Y.; Zheng, J.J.; Tang, J.; Huang, C.Z. Visual observation of the mercury-stimulated peroxidase mimetic activity of gold nanoparticles. Chem. Commun. 2011, 47, 11939-11941. [CrossRef] [PubMed]

12. Cai, S.; Lao, K.; Lau, C.; Lu, J. “Turn-On” Chemiluminescence Sensor for the Highly Selective and Ultrasensitive Detection of $\mathrm{Hg}^{2+}$ Ions Based on Interstrand Cooperative Coordination and Catalytic Formation of Gold Nanoparticles. Anal. Chem. 2011, 83, 9702-9708. [CrossRef] [PubMed]

13. Song, C.; Yang, B.; Yu, Z.; Yang, Y.; Wang, L. Ultrasensitive sliver nanorods array SERS sensor for mercury ions. Biosens. Bioelectron. 2017, 15, 59-65. [CrossRef] [PubMed]

14. He, Z.-J.; Kang, T.-F.; Lu, L.-P.; Cheng, S.-Y. An electrochemiluminescence sensor based on CdSe@CdSfunctionalized MoS2 and a GOD-labeled DNA probe for the sensitive detection of $\mathrm{Hg}(\mathrm{ii})$. Anal. Methods 2020, 12, 491-498. [CrossRef]

15. Long, F.; Zhu, A.; Shi, H. Recent Advances in Optical Biosensors for Environmental Monitoring and Early Warning. Sensors 2013, 13, 13928-13948.

16. Tan, L.; Zhang, Y.; Qiang, H.; Li, Y.; Sun, J.; Hu, L.; Chen, Z. A sensitive Hg(II) colorimetric sensor based on synergistic catalytic effect of gold nanoparticles and Hg. Sens. Actuator B Chem. 2016, 229, 686-691. [CrossRef]

17. Peng, C.-F.; Pan, N.; Xie, Z.-J.; Wu, L.-L. Highly sensitive and selective colorimetric detection of $\mathrm{Hg}^{2+}$ based on the separation of $\mathrm{Hg}^{2+}$ and formation of catalytic DNA-gold nanoparticles. Anal. Methods 2016, 8, 1021-1025. [CrossRef] 
18. Martinez, A.W.; Phillips, S.T.; Whitesides, G.M. Three-dimensional microfluidic devices fabricated in layered paper and tape. Proc. Natl. Acad. Sci. USA 2008, 105, 19606-19611. [CrossRef]

19. Fu, E.; Downs, C. Progress in the development and integration of fluid flow control tools in paper microfluidics. Lab Chip 2017, 17, 614-628. [CrossRef]

20. Nilghaz, A.; Lu, X. Detection of antibiotic residues in pork using paper-based microfluidic device coupled with filtration and concentration. Anal. Chim. Acta 2019, 1046, 163-169. [CrossRef]

21. Pena-Pereira, F.; Lavilla, I.; Bendicho, C. Paper-based analytical device for instrumental-free detection of thiocyanate in saliva as a biomarker of tobacco smoke exposure. Talanta 2016, 147, 390-396. [CrossRef] [PubMed]

22. Feng, L.; Li, X.; Li, H.; Yang, W.; Chen, L.; Guan, Y. Enhancement of sensitivity of paper-based sensor array for the identification of heavy-metal ions. Anal. Chim. Acta 2013, 780, 74-80. [CrossRef] [PubMed]

23. Evans, E.; Moreira Gabriel, E.F.; Benavidez, T.E.; Tomazelli Coltro, W.K.; Garcia, C.D. Modification of microfluidic paper-based devices with silica nanoparticles. Analyst 2014, 139, 5560-5567. [CrossRef] [PubMed]

24. Jeong, S.-G.; Lee, S.-H.; Choi, C.-H.; Kim, J.; Lee, C.-S. Toward instrument-free digital measurements: A three-dimensional microfluidic device fabricated in a single sheet of paper by double-sided printing and lamination. Lab Chip 2015, 15, 1188-1194. [CrossRef] [PubMed]

25. Yetisen, A.K.; Akram, M.S.; Lowe, C.R. Paper-based microfluidic point-of-care diagnostic devices. Lab Chip 2013, 13, 2210-2251. [CrossRef] [PubMed]

26. Liu, H.; Crooks, R.M. Three-Dimensional Paper Microfluidic Devices Assembled Using the Principles of Origami. J. Am. Chem. Soc. 2011, 133, 17564-17566. [CrossRef]

27. Ishii, S.; Segawa, T.; Okabe, S. Simultaneous Quantification of Multiple Food- and Waterborne Pathogens by Use of Microfluidic Quantitative PCR. Appl. Environ. Microbiol. 2013, 79, 2891-2898. [CrossRef]

28. Ornatska, M.; Sharpe, E.; Andreescu, D.; Andreescu, S. Paper Bioassay Based on Ceria Nanoparticles as Colorimetric Probes. Anal. Chem. 2011, 83, 4273-4280. [CrossRef]

29. Lee, Y.-F.; Huang, C.-C. Colorimetric Assay of Lead Ions in Biological Samples Using a Nanogold-Based Membrane. ACS Appl. Mater. Interfaces 2011, 3, 2747-2754. [CrossRef]

30. Ratnarathorn, N.; Chailapakul, O.; Henry, C.S.; Dungchai, W. Simple silver nanoparticle colorimetric sensing for copper by paper-based devices. Talanta 2012, 99, 552-557. [CrossRef]

31. Chaiyo, S.; Siangproh, W.; Apilux, A.; Chailapakul, O. Highly selective and sensitive paper-based colorimetric sensor using thiosulfate catalytic etching of silver nanoplates for trace determination of copper ions. Anal. Chim. Acta 2015, 866, 75-83. [CrossRef] [PubMed]

32. Esmaeili, N.; Rakhtshah, J.; Kolvari, E.; Shirkhanloo, H. Ultrasound assisted-dispersive-modification solid-phase extraction using task-specific ionic liquid immobilized on multiwall carbon nanotubes for speciation and determination mercury in water samples. Microchem. J. 2020, 154, 104632. [CrossRef]

33. Figueredo, F.; Garcia, P.T.; Corton, E.; Coltro, W.K.T. Enhanced Analytical Performance of Paper Microfluidic Devices by Using Fe3O4 Nanoparticles, MWCNT, and Graphene Oxide. ACS Appl. Mater. Interfaces 2016, 8, 11-15. [CrossRef]

34. Wang, P.; Ge, L.; Yan, M.; Song, X.; Ge, S.; Yu, J. Paper-based three-dimensional electrochemical immunodevice based on multi-walled carbon nanotubes functionalized paper for sensitive point-of-care testing. Biosens. Bioelectron. 2012, 32, 238-243. [CrossRef] [PubMed]

35. He, Y.; Zhang, S.; Zhang, X.; Baloda, M.; Gurung, A.S.; Xu, H.; Zhang, X.; Liu, G. Ultrasensitive nucleic acid biosensor based on enzyme-gold nanoparticle dual label and lateral flow strip biosensor. Biosens. Bioelectron. 2011, 26, 2018-2024. [CrossRef] [PubMed]

36. Qiao, Y.; Shang, J.; Li, S.; Feng, L.; Jiang, Y.; Duan, Z.; Lv, X.; Zhang, C.; Yao, T.; Dong, Z. Fluorimetric Mercury Test Strips with Suppressed "Coffee Stains" by a Bio-inspired Fabrication Strategy. Sci. Rep. 2016, 6, 36494. [CrossRef]

37. Zhang, Y.; Zuo, P.; Ye, B.C. A low-cost and simple paper-based microfluidic device for simultaneous multiplex determination of different types of chemical contaminants in food. Biosens. Bioelectron. 2015, 68, 14-19. [CrossRef]

38. Li, D.; Sun, Y.; Shen, Q.; Zhang, Q.; Huang, W.; Kang, Q.; Shen, D. Smartphone-based three-channel ratiometric fluorescent device and application in filed analysis of $\mathrm{Hg}^{2+}, \mathrm{Fe}^{3+}$ and $\mathrm{Cu}^{2+}$ in water samples. Microchem. J. 2020, 152, 104423. [CrossRef] 
39. Zhou, T.; Liu, J.-J.; Xu, Y.; Wu, Z.-Y. Fast and sensitive screening detection of tetracyclines with a paper-based analytical device. Microchem. J. 2019, 145, 703-707. [CrossRef]

40. Han, K.N.; Choi, J.S.; Kwon, J. Gold nanozyme-based paper chip for colorimetric detection of mercury ions. Sci. Rep. 2017, 7, 7. [CrossRef]

41. Xie, Z.-J.; Bao, X.-Y.; Peng, C.-F. Highly Sensitive and Selective Colorimetric Detection of Methylmercury Based on DNA Functionalized Gold Nanoparticles. Sensors 2018, 18, 2679. [CrossRef] [PubMed]

42. Mei, Z.; Chu, H.; Chen, W.; Xue, F.; Liu, J.; Xu, H.; Zhang, R.; Zheng, L. Ultrasensitive one-step rapid visual detection of bisphenol A in water samples by label-free aptasensor. Biosens. Bioelectron. 2013, 39, 26-30. [CrossRef] [PubMed]

Publisher's Note: MDPI stays neutral with regard to jurisdictional claims in published maps and institutional affiliations.

(C) 2020 by the authors. Licensee MDPI, Basel, Switzerland. This article is an open access article distributed under the terms and conditions of the Creative Commons Attribution (CC BY) license (http://creativecommons.org/licenses/by/4.0/). 